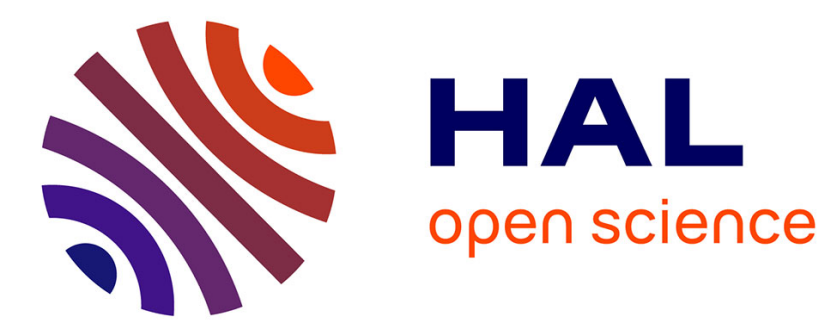

\title{
The OPERA experimental program with CNGS
}

D. Duchesneau

\section{To cite this version:}

D. Duchesneau. The OPERA experimental program with CNGS. International Workshop on Topics in Astroparticle and Underground Physics 8 TAUP2003, Sep 2003, Seattle, United States. pp.411-414, 10.1016/j.nuclphysbps.2004.11.094 . in2p3-00014170

\section{HAL Id: in2p3-00014170 https://hal.in2p3.fr/in2p3-00014170}

Submitted on 25 Nov 2003

HAL is a multi-disciplinary open access archive for the deposit and dissemination of scientific research documents, whether they are published or not. The documents may come from teaching and research institutions in France or abroad, or from public or private research centers.
L'archive ouverte pluridisciplinaire HAL, est destinée au dépôt et à la diffusion de documents scientifiques de niveau recherche, publiés ou non, émanant des établissements d'enseignement et de recherche français ou étrangers, des laboratoires publics ou privés. 
LAPP-EXP 2003-25

November 2003

The OPERA experimental program with CNGS

D. Duchesneau

LAPP-IN2P3-CNRS

9 chemin de Bellevue - BP. 110

F-74941 Annecy-le-Vieux Cedex

Invited talk at the 8th International Workshop on Topics in Astroparticle and Underground Physics (TAUP2003), Seattle, Etats-Unis, September 5-9, 2003 


\title{
The OPERA Experimental Program with CNGS
}

\author{
D. Duchesneau ${ }^{\mathrm{a}}$ *
}

\section{${ }^{a}$ LAPP, IN2P3-CNRS,}

9 Chemin de Bellevue, BP110, F-74941, Annecy-le-Vieux, France

(email: duchesneau@lapp.in2p3.fr)

This paper reviews the current status of the OPERA detector preparation which is designed to investigate the neutrino oscillation properties using the future CERN neutrino beam called CNGS. The physics potential and performances for neutrino oscillation studies including $\nu_{\mu} \rightarrow \nu_{e}$ search will also be presented.

\section{Introduction}

OPERA [1] is a first generation long baseline neutrino experiment to be located in the Gran Sasso underground laboratory. The detector is a massive hybrid detector with nuclear emulsions used as very precise tracking devices and electronic detectors to locate the neutrino interaction events in the emulsions.

It is designed to primarily search for $\nu_{\tau}$ appearance in the CERN high energy $\nu_{\mu}$ beam CNGS [2] at $730 \mathrm{~km}$ from the neutrino source, in order to establish unambiguously the origin of the neutrino oscillations observed at the atmospheric $\Delta m^{2}$ scale. The best fit of the $\nu_{\mu} \rightarrow \nu_{\tau}$ oscillation hypothesis to the Super-Kamiokande atmospheric data gives the oscillation parameters $\Delta m^{2}=2.0 \times 10^{-3} \mathrm{eV}^{2}$ with $\sin ^{2} 2 \theta=1.0$ [3]. The range of allowed values at $90 \%$ CL correspond to $1.3 \times 10^{-3}<\Delta m^{2}<3.0 \times 10^{-3} \mathrm{eV}^{2}$ and $\sin ^{2} 2 \theta>0.90$.

The CNGS neutrino beam is a high energy beam optimised for $\nu_{\tau}$ appearance with a mean neutrino energy of about $17 \mathrm{GeV}$. The beamline characteristics are summarised in Ref. [4] and the construction status of the CNGS project can be found in Ref. [5]. The possibility of an increase of the neutrino beam intensity by a factor $1.5 \mathrm{com}-$ pared to the original design is under study [6]. However the feasability for this upgrade should await results of tests performed in 2003 .

Using the CERN SPS accelerator in a shared

\footnotetext{
* Representing the OPERA Collaboration.
}

mode, $6.76 \times 10^{19}$ protons on target (pot) can be delivered during one year, assuming 200 days of operation. The number of charged current and neutral current interactions expected in the Gran Sasso laboratory from $\nu_{\mu}$ are about 4000 /kton/year and 1240 /kton/year respectively. If the $\nu_{\mu} \rightarrow \nu_{\tau}$ oscillation hypothesis is confirmed, the number of $\tau$ 's produced via charged current interaction at the Gran Sasso should be of the order of $16 / \mathrm{kton} /$ year for $\Delta m^{2}=2.0 \times 10^{-3} \mathrm{eV}^{2}$ at full mixing.

\section{Status of the detector construction}

The $\nu_{\tau}$ appearance search is based on the observation of events produced by charged current interaction (CC) with the $\tau$ decaying in all possible decay modes. The principle of the OPERA experiment is to observe the $\tau$ trajectories and the decay products in emulsion films composed of two thin emulsion layers ( $50 \mu \mathrm{m}$ thick) put on either side of a plastic base (200 $\mu \mathrm{m}$ thick). To achieve a large target mass, the emulsion films are interleaved with $1 \mathrm{~mm}$ thick lead plates. The basic detector unit, called ECC brick, is obtained by stacking 56 lead plates with 57 emulsion films. In order to reach 1.8 kton target mass, 206336 bricks will be installed into walls separated from each other by vertical planes of electronic target trackers. A more complete description of the detector setup can be found in Ref [4].

A brief summary of the construction status of the different detector parts follows. 


\subsection{The Emulsion Films and Lead}

The emulsion films are produced in Japan by FUJI company and the mass production started in April 2003 at a rate of about $8000 \mathrm{~m}^{2} /$ month. The entire production will correspond to a total of $150000 \mathrm{~m}^{2}$. To erase the cosmic ray tracks recorded during film production and subsequent manipulation, a refreshing process, consisting of leaving the emulsion in a $95 \%$ relative humidity during 3 days at a temperature of $30^{\circ} \mathrm{C}$, is performed in the Tono mine in Japan. This procedure allows to erase more than $98 \%$ of the tracks before transportation to Gran Sasso.

The lead used for the bricks consists of low radioactivity lead (Boliden) with $0.7 \% \mathrm{Ca}$ added for rigidity purpose. The lead thickness is controlled with a $10 \mu \mathrm{m}$ accuracy. A prototype production is scheduled with the Goslar firm in Germany.

\subsection{The Target Tracker}

The electronic target tracker is composed of $\mathrm{X}$ and Y planes of 256 AMCRYS-H plastic scintillator strips $(6.7 \mathrm{~m} \mathrm{x} 2.5 \mathrm{~cm} \times 1 \mathrm{~cm})$. Each plane is divided in 4 modules. Each strip is read out on both ends by a Kuraray wave length shifting optical fiber connected to 64 channel Hamamatsu photomultiplier tubes. The main goal of the electronic detector is to provide a trigger with an efficiency greater than $99 \%$ for neutrino interactions and an efficient localisation of the bricks where the events occur.

The construction and assembly of the target tracker modules take place in Strasbourg. The production rate should be of 8 modules per week. Delivery and installation at the Gran Sasso laboratory is planned to start beginning of 2004 .

\subsection{The Muon spectrometer}

The muon spectrometer allows a determination of the charge and momentum of muons going through by measuring their curvature in a dipolar magnet which provides 1.6 Tesla transverse to the neutrino beam axis. Each spectrometer is equipped with six vertical planes of drift tubes as precision tracker together with 22 planes $(8 \mathrm{x} 8$ $\mathrm{m}^{2}$ ) of RPC bakelite chambers. The RPC's are located in the magnets between the $5 \mathrm{~cm}$ thick vertical iron slabs. The installation of the final spectrometers in the Hall C of the Gran Sasso underground laboratory has already started since May 2003. The iron slabs, the yokes, the coils and the power supplies have been ordered in the mean time. The first batch of RPC chambers are expected to arrive in November 2003 at Gran Sasso to be installed in the first spectrometer.

The precision tracker planes are composed of 4 staggered layers of 168 aluminium tubes, $8 \mathrm{~m}$ long with $38 \mathrm{~mm}$ outer diameter. A full scale prototype module has been built and tested in Hamburg. The spatial resolution of this detector is better than $500 \mu \mathrm{m}$.

The physics performance of the complete spectrometer reduces the charge confusion to less than $0.3 \%$ and gives a momentum resolution better than $20 \%$ for momentum less than $50 \mathrm{GeV}$. The muon identification efficiency reaches $95 \%$ using the target tracker information for the cases where the muons stop inside the target.

\section{Physics performance: $\nu_{\mu} \rightarrow \nu_{\tau}$ search}

The $\tau$ decay channels investigated by OPERA are the e, $\mu$ and hadron. Table 1 summarises the OPERA performance after 5 years of running with the $50 \%$ increased CNGS intensity $\left(3.35 \times 10^{20} \mathrm{pot}\right)$. The numbers in parenthesis are obtained from the nominal CNGS intensity delivering $2.25 \times 10^{20}$ pot in 5 years. The number of expected signal events from $\nu_{\mu} \rightarrow \nu_{\tau}$ oscillation is given as a function of the studied channel for three different values of $\Delta m^{2}$ at full mixing. The total efficiency including the branching ratios amounts to $9.1 \%$ and the total background is estimated to be less than 1.06 event. The main background sources are charm decays (54\%), large angle muon scattering (16\%) and hadron reinteractions (29\%). A probability larger than $95 \%$ to have a $4 \sigma$ significant effect after 5 years is reached for $\Delta m^{2}>2.0 \times 10^{-3} \mathrm{eV}^{2}$. This probability drops to $45 \%$ for $\Delta m^{2}=1.3 \times 10^{-3} \mathrm{eV}^{2}$.

\section{Search for $\nu_{\mu} \rightarrow \nu_{e}$ appearance}

Having excellent electron identification capabilities, OPERA has estimated its sensitivity in searching for $\nu_{\mu} \rightarrow \nu_{e}$ appearance [7] with the 
Table 1

Summary of the expected numbers of $\tau$ events in 5 years for different $\Delta m^{2}$ with the expected background and detection efficiencies per decay channel for OPERA. The numbers in parenthesis are obtained with the initial CNGS intensity design

\begin{tabular}{lccccc}
\hline channel & \multicolumn{3}{c}{ signal for $\Delta m^{2}\left(\mathrm{eV}^{2}\right)$} & $\epsilon \mathrm{xBr}$ & Background \\
& $1.3 \times 10^{-3}$ & $2.0 \times 10^{-3}$ & $3.0 \times 10^{-3}$ & & \\
\hline$\tau \rightarrow \mathrm{e}$ & $1.8(1.2)$ & $4.1(2.7)$ & $9.2(6.1)$ & $3.4 \%$ & $0.31(0.21)$ \\
$\tau \rightarrow \mu$ & $1.4(0.9)$ & $3.4(2.3)$ & $7.6(5.1)$ & $2.8 \%$ & $0.33(0.22)$ \\
$\tau \rightarrow \mathrm{h}$ & $1.5(1.0)$ & $3.5(2.3)$ & $7.8(5.2)$ & $2.9 \%$ & $0.42(0.28)$ \\
\hline Total & $4.7(3.1)$ & $11.0(7.3)$ & $24.6(16.4)$ & $9.1 \%$ & $1.06(0.71)$ \\
\hline
\end{tabular}

CNGS beam. The analysis principle is based on a search for an excess of $\nu_{e} \mathrm{CC}$ events at low neutrino energies. The main background comes from the electron neutrino contamination $(0.8 \%)$ present in the beam. The analysis takes into account the electron events coming from $\nu_{\mu} \rightarrow \nu_{\tau}$ events where $\tau \rightarrow e \nu_{\tau} \nu_{e}$ since both oscillations would occur at the atmospheric $\Delta m^{2}$ scale. These events distort the kinematical distributions where the low energy events contribute. The sensitivity to $\theta_{13}$ is obtained by doing a $\chi^{2}$ minimisation using the visible energy, the missing transverse energy and the electron energy distributions in which the oscillation parameter varies. Table 2 summarises the expected number of selected events, assuming 5 years running and still the original CNGS design intensity $\left(2.25 \times 10^{20}\right.$ pot), from $\nu_{\mu} \rightarrow \nu_{e}$ and $\nu_{\mu} \rightarrow \nu_{\tau}$ oscillations and from background $\nu_{e}$ at 3 values of $\theta_{13}$. Background events coming from $\nu_{\mu} \mathrm{CC}$ and $\nu_{\mu} \mathrm{NC}$ interactions amount to 1.0 and 5.2 respectively.

Table 2

Expected number of signal and background events in 5 years obtained in the search of $\nu_{\mu} \rightarrow \nu_{e}$ oscillation at $\Delta m^{2}=2.5 \times 10^{-3} \mathrm{eV}^{2}$.

\begin{tabular}{ccccc}
$\begin{array}{c}\theta_{13} \\
(\mathrm{deg})\end{array}$ & $\sin ^{2} 2 \theta_{13}$ & $\nu_{e} \mathrm{CC}$ & $\begin{array}{c}\nu_{\mu} \rightarrow \nu_{\tau} \\
\tau \rightarrow \mathrm{e}\end{array}$ & $\begin{array}{c}\text { signal } \\
\nu_{\mu} \rightarrow \nu_{e}\end{array}$ \\
\hline 9 & 0.095 & 18 & 4.5 & 9.3 \\
7 & 0.058 & 18 & 4.6 & 5.8 \\
5 & 0.030 & 18 & 4.6 & 3.0 \\
\hline
\end{tabular}

The limit obtained by OPERA at $90 \% \mathrm{CL}$ on $\theta_{13}$ is $7.1^{\circ}$ after 5 years, which leads to significant improvement over the actual CHOOZ limit [8] and opens an important window on the third mixing angle.

\section{Conclusion}

The CNGS construction is progressing well. The project is on schedule and a startup is expected for June 2006. At the same time OPERA enters the construction phase and should be ready to take data by 2006 .

The detector performances are such that the first evidence for $\nu_{\mu} \rightarrow \nu_{\tau}$ appearance signal could be seen after only a few years of data taking. The very good electron identification and measurement of the detector give the possibility to explore the $\nu_{\mu} \rightarrow \nu_{e}$ appearance channel pushing down the $\theta_{13}$ limit.

\section{REFERENCES}

1. OPERA Collaboration, M. Guler et al., Experiment proposal, CERN-SPSC-2000-028 and LNGS P25/2000 (2000); Status Report on the OPERA experiment, CERN-SPSC2001-025 and LNGS-EXP 30/2001 (2001).

2. G. Acquistapace et al., CERN 98-02 and INFN/AE-98/105 (1998); R. Bailey et al., "Addendum to Report CERN 98-02", CERNSL 99-034 and INFN/AE-99/05 (1999); A.E. Ball et al., SL-Note 2000-063 (2000).

3. Y. Hayato, contribution to the EPS conference, july 2003, Aachen, Germany.

4. D. Duchesneau, Nucl. Phys. B (Proc. Suppl.) 123 (2003) 279 [hep-ex/0209082].

5. F. Arneodo, these proceedings.

6. R. Cappi et al., CERN-SL-2001-032 (2001).

7. M. Komatsu, P. Migliozzi and F. Terranova, J. Phys. G29 (2003) 443 [hep-ph/0210043].

8. CHOOZ Collaboration, M. Apollonio et al., Phys. Lett. B 466 (1999) 415. 